\title{
Presentación de candidatos a la decanatura 1995-1998
}

NOMBRE: NICOLAS A. NUÑEZ LEMA: Por una Facultad del siglo XXI

Formación académica:

'- Psicólogo, U. INCCA de Co-lombia, 1986

- Especialista en psicología de la salud. Universidad Nacional de Colombia, 1990.

- Maestría en Filosofia, U. INCCA de Colombia, 1988 graduado con mención honorífica.

Experiencia académica:

- Profesor tiempo completo, Universidad Surcolombiana desde 1991 hasta la fecha.

- Profesor Universidad SurcolombianaCINDE, dedicación parcial.

- Profesor de tiempo completo. Universidad INCCA de Colombia, Coordinador área metodología de investigación psicológica.

- Asistente Centro de Investigaciones de Psicología.

- Director Laboratorio de Psicología.

- Coordinador proyecto en inteligencia artificial, de enero 1988 hasta abril de 1991.

Conferencias dictadas::

CONGRESO COLOMBIANO DE PSICOLOGIA. Medición del estrés en trabajadores estatales y privados en el departamento del Huila, Bogotá, agosto de 1993.

- CONGRESO COLOMBIANO DE PSICOLOGIA. Aportes de la teoria conductual en el manejo del dolor crónico. Pereira, 1991.

- VIII Encuentro de Facultades de Educación del suroccidente Colombiano. Fundamentos teóricos sobre la práctica comunitaria, Neiva, 1991.
- Asociación Colombiana para el avance de la Psicología. Inteligencia humana y sus aportes a la inteligencia artificial.

Proyectos de la Facultad de Educación:

- Implementación de un currículo de Informática Educativa y Matemática en la Universidad Surcolombiana, en colaboración con los profesores Helga Truque y Mauro Montealegre.

- Implementación del Programa de pregrado en Psicología.

Implementación y dirección de la Especialización en Desarrollo Humano y Educación Sexual, en colaboración con la profesora Margarita Chacón.

- Profesor asistente en la cátedra de Desarrollo Humano en la Maestría en Educación y Desarrollo ComunitarioCINDE -USCO.

Publicaciones realizadas::

- EVALUACIÓN DEL DESEMPEÑO. Neiva, USCO, 1994. acompañado con software educativo.

- Currículo de informática educativa para la Facultad de Educación. In Asociación de Egresados en Administradores Educativos, Neiva. 1993.

$-0-0-0-0-0-0$

NOMBRE: MARCELINO J. COLUNJE

LEMA: "Por una facultad para la investigación y la cultura."

Formación académica-pedagógica: 
- Normalista Superior. Normal de Pasto.

- Licenciado en Matemáticas y Física. Universidad.de Tunja.

- Magister en Admón. Educativa. Universidad del Valle.

- Curso de Especialización en Curriculum. Universidad de Puerto (Puerto Rico (EE.UU.)

- Curso de Especialización en Física del Valle.

- Curso de Especialización en CurriculumUniversidad de Puerto'Puerto Rico (EE.UU.)

- Curso de Especialización en FísicaUniversidad del Valle.

\section{Experiencia docente-administrativa}

- Profesor Primaria Col. San Juan Bosco de Pasto.

- Profesort Secundaria Colegio de Popayán

- Supervisor Secreeducación Cauca.

- Jefe Educación Media Secreeducació Cauca.

- Catedrático Universidad del Cauca.

- Rector Colegio de Santa Librada, Neiva -Jefe de Programa Admón. Educati-va Universidad Surcolombiana, Neiva.USCO. - Jefe Dpto. Educació. USCO.

- Profesor visitante (Postgrado) Universidad del Valle.

- Coordinador Postgrado Admón. de la Educación. Convenio USCO. Univalle.

Investigaciones $\mathbf{y}$ asesorias:

- Pertil del docente del Huila. 1a. parte.

- Estudio de factibilidad Escuela de Artes del Huila.

- Reseña histórica del sindicalismo del magisterio huilense.

- Implementación del bachillerato agricola de la cárcel judicial de Neiva.

- Plan de Desarrollo Educativo del Colegio Nacional Santa Librada de Neiva, hacia la celebración de sus 150 años de fundación.

- Proyecto de creación colegio privado FEDUSCO.

- Proyecto Centro de reforón colegio privado FEDUSCO.
Proyecto Centro de reforzamiento académico para estudiantes de colegios oficiales de Neiva.

\section{Representaciones:;}

- Representante de los profesores ante el Consejo Superior, Consejo Académico, Comité de Selección y Comité de Bienestar Estudiantil.

\section{$-0-0-0-0-0-$}

\section{Nomrbre: LUIS EVELIO VANEGAS R}

\section{-Formación académica:}

- Licenciado en Idiomas Inglés-Francés. Universidad Nacional de Colombia de Bogotá, 1973.

- Especialización "Diploma in the Teaching of Overseas" Leeds University Leeds Inglaterra 1978-1979.

- COFE-ELTO Postgraduate Course'Thames Valley University-Londres, 1992.

Experiencia docente::

- Universidad Surcolombiana, profesor de tiempo completo desde 1975 hasta la presente. Actualmente profesor titular adscrito a la Facultad de Educación Programa de Lenguas Modernas.

- Colegio Nacional Santa Librada. Neivaprofesor de inglés, 1974-1977.

- Hankuk University. Seúl-Corea del Sur ,profesor de Español, enero-diciembre de 1980.

- Korean Broadcasting Center. Profesor de Español a través de televisión. Mayodiciembre de 1980. Seúl Corea del Sur.

\section{Publicaciones realizadas::}

Análisis fonológico del guambianomimeografiado, 1973. Coautor.

- Analicemos y discutamos para estudiantes Coreanos del nivel intermedio $y$ avanzado de Español. Libro-Corea-1980. Coautor.

- Análisis de la situación actual de la enseñánzaq del inglés y del francés en el Huila. Investigación, 1987. Autor. 
- A proposal of a Framework for the Teachin of Englis in the Colombia B.A. Programas Londres 1992. Coautor (Propuesta de reforma Curricular a las Licenciaturas en Idiomas para las Universidades Estatales.

\section{Otras actividades curriculares:}

- Presidente de la Asociación Colombiana de Profesores de Enseñanza Secundaria ACPES-Huila, 1975.

- Presidente de la Asociación Sindical de Profesores Universitarios ASPU-Huila, 1977 '1978.

- Representante de los profesores al Consejo Supèrior de la Universidad Surcolombiana, 1987.

- Primer presidente de la Asociació Colombiana de Profesores de Inglés ASOCOPI- Huila, 1990

- Coordinador del Proyecto COFEELTO Universidad Surcolombiana, 1992 hasta la presente.

- Coinvestigador principal. La enseñanza del Idioma Inglés en la Educación Básica Primaria en el municipio de Neiva. Investigación en proceso.

- Miembro de la comisión de Reestructuración de la Facultad de Educació de la Universidad Surcolombiana, desde el 22 de noviembre de 1994.-

\section{Ultima ponencia:}

- Implicaciones de la Ley 115 de 1994 para la Facultad de Educación. Encuentro Nacional de Facultad de Educación., Medellín, octubre de 1994.

$$
-0-0-0-0
$$

Nombre: DAVID PLATA:

Edad: 38 años

Profesión: Sociólogo

Especialista en Regional.

Gestión del Desarrollo

Estudios complementarios:

- Creatividad comunitaria

- Interdisicplina
- Desarrollo Comunitario

- Desarrollo empresarial

- Sicología

- Cibernética social

- Formación pedagógica SENA

Participación y dirección de evemtos en:

- Acción Comunal

- Vivienda popular

- Sociología

- Microempresas

. Organizaciones sociales

- Desarrollo Comunitario

- Descentralización municipal

- Planeación y gestión

- Participación y democracia

- Organización profesional

Miembro de la Asociación interdisicplinaria Colombiana -ASIC- y de la International Social Cybernetics Association-ISCA- así como de la Fundación para el Desarrollo de la Universidad SurcolombianaFUNDAUSCO-y de la Asociación Sindical de Profesores Universitarios.

- Miembro de diversas organizaciones sociales y comunitarias y de las Juntas Directivas de la Empresa de Vivienda Popular de Neiva -EMVINEIVA- y el Instituto Huilense de Cultura.

Experiencia curricular:

- Profesor de secundaria

- Asesor de proyectos de Desarrollo Comunitario.

- Profesor catedrático de la universidad Surcolombiana 1984 y 1989, en las áreas de Socoedad y Salud. Sociología Rural, Sociología de las Organizaciones, Sociología de la Administración y Sociologia Educativa.

- Profesor catedrático de la Universidad Antonio Nariño en Comunicación y procesos de grupo en 1987.

- Instructor de Desarrollo Social Empresarial, SENA regional Huila durante 8 años. 
- Coordinador Unidad de Vivienda Popular de Neiva, y Jefe de la Divisón de Desarrollo Social de la Empresa de Vivienda Popular de Neiva entre 1966 y 1987.

- Profesional de la sección de Servicios Técnicos del ICBF Regional Huila, entre 1987 y enero de 1990.

- Director del Postgrado de Especialización en Gestión del Desarrollo Regional de la Universidad Surcolombiana, entre junio de 1993 y mayo de 1994.

\section{Proyectos de investigación:}

- Habilidad y Desarrollo Integral -CESCOUSCO-1964.

- Plan Integral de Desarrollo de Neiva, 'PIDNE, primera y segunda etapas 19841985, CIDEC-USCO.

- Censo de la Industria Manufacturera de Neiva CIDEC-USCO, 1984.

- Precenso poblacional urbano, CIDECUSCO, 1985.

- Estduio socio-económico y ambiental del impacto de la represa de Betania, USCO, CHB, 1991.

- Proyectos de Desarrollo InstitucionaklPDI- en los municipios de Palestina, Baraya, San Agustín y Santa María, 1994.

- Ha escrito varios ensdayos y trabajos en áreas de desarrollo comunitario y desarrollo social, y documentos sobre análisis de contexto y vida universitaria.

- Profesor de tiempo completo de la Universidad Surcolombiana, adscrito a la Facultad de Educación, Departamento. de Psicopedagogía, desde enero 12 de 1990.
Nombre; HIPOLITO CAMACHO COY

Lema; COMPROMISO ACADEMICO CON INDEPENDENCIA POLITICA.

\section{Formación académica:}

- Licenciado én Educación FísicaUniversidad Surcolombiana-Neiva.

- Magister en Desarrollo Educativo y Social -CINDE- Universidad Pedagógica Nacional.

\section{Experiencia docente:}

- Profesor de básica primaria 1973-976

- Profesor de educación secundaria 19761983

- Profesor universitario en las modalidades presencial y a distancia, niveles de pregrado y postgrado, 1983-1995.

\section{Investigaciones:}

- Diseño de programas de Educación Física para la escuela primaria, 1985

- Alternativa curricular de la Educación Física para secundaria, 1991.

- Premio Nacional de Investigación en Ciencias del Deporte, Educación Física, 1994.

\section{Presentaciones:}

- Jefe de Programa de Educación Física, 1990-1991.

- Coordinador Práctica Profesional Facultad de Educación, 1992-1993

- Coordinador Comité Investigaciones de la Facultad de Educación, 1994. 